\title{
A Review on Bioanalytical Method Development and Various Validation Stages Involved In Method Development Using RP- HPLC
}

\author{
Imran N. Mujewar¹, Omprakash G. Bhusnure1*, Sneha R.Jagtap, Sachin B. Gholve1, Padmaja S. Giram², Atul \\ B. Savangikar ${ }^{3}$
}

\author{
${ }^{1}$ Department of Quality Assurance, Channabasweshwar Pharmacy College, Latur, Maharashtra, India. \\ 2 Department of Pharmacology, Channabasweshwar Pharmacy College, Latur, Maharashtra, India.
}

3 FDC Pharmaceutical Ltd, DN Nagar, Andheri, Mumbai.

\section{ABSTRACT}

Bioanalysis is an essential part in drug discovery and development. Bioanalysis is related to the analysis of analytes (drugs, metabolites, biomarkers) in biological samples and it involves several steps from sample collection to sample analysis and data reporting. The first step is sample collection from clinical or preclinical studies; then sending the samples to laboratory for analysis. Second step is sample preparation and it is very important step in bioanalysis. In order to reach reliable results, a robust and stable sample preparation method should be applied. The role of sample preparation is to remove interferences from sample matrix and improve analytical system performance. Sample preparation is often labour intensive and time consuming. This guideline defines key elements necessary for the validation of bioanalytical methods. The guideline focuses on the validation of the bioanalytical methods generating quantitative concentration data used for pharmacokinetic and toxicokinetic parameter determinations. Guidance and criteria are given on the application of these validated methods in the routine analysis of study samples from animal and human studies. Measurement of drug concentrations in biological matrices (such as serum, plasma, blood, urine, and saliva) is an important aspect of medicinal product development. It is therefore paramount that the applied bioanalytical methods used are well characterised, fully validated and documented to a satisfactory standard in order to yield reliable re sults. This review provides an overview of bioanalytical method development and validation and main principles of method validation stages discussed.
\end{abstract}

Keywords: Bioanalysis, Sample Preparation, Bioanalytical Method Development and Validation

Article Info: Received 11 June 2019; $\quad$ Review Completed 08 August 2019; $\quad$ Accepted 17 August $2019 ; \quad$ Available online 25 August 2019

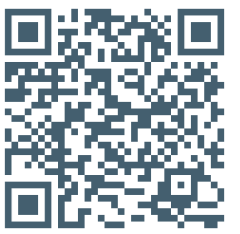

Cite this article as:

Mujewar IN, Bhusnure OG, Jagtap SR, Gholve SB, Giram PS, Savangikar AB, A Review on Bioanalytical Method Development and Various Validation Stages Involved In Method Development Using RP - HPLC , Journal of Drug Delivery and Therapeutics. 2019; 9(4-s):789-795 http://dx.doi.org/10.22270/jddt.v9i4-s.3422

Omprakash G. Bhusnure, Department of Pharmacology, Channabasweshwar Pharmacy College, Latur, Maharashtra, India.

\section{INTRODUCTION}

Measurement of drug concentrations in biological matrix (such as serum, plasma, blood, urine, and saliva) is an essential aspect of medicinal product development. Such data may be required to support applications for new actives substances and generics as well as variation to authorised drug products. The results of animal toxicokinetic studies and of clinical trials, including bioequivalence studies are used to make significant decisions supporting the safety and efficacy of a medicinal drug substance or product. Bioanalysis is not only measuring of small molecules such as drugs and metabolites but also to identify large molecules such as proteins and peptides. Bioanalysis is well established in pharmaceutical companies to support drug discovery and drug development. Bioanalysis has an important role to perform the toxicokinetic (TK), pharmacokinetic (PK) and pharmacodynamics (PD) studies of new drugs. It is therefore principle that the applied bioanalytical methods used are well characterised, fully validated and documented to a satisfactory standard in order to yield reliable results. A bioanalytical method is a set of all of the procedures involved in the collection, processing, storage or storing and analysis of a biological matrix for an analyte. Analytical methods employed for quantitative determination of drugs and their metabolites in biological fluids are the key determinants in generating reproducible and reliable data that in turn are used in the evaluation and interpretation of bioavailability, bioequivalence and pharmacokinetics. Method development is a scientific procedure. It involves evaluation and optimization of the various stages of sample preparation, chromatographic conditions, detection and quantification. [1-10] 
The bioanalysis procedure includes sampling, sample preparation, analysis, calibration and data evaluation and reporting (Fig.1). In modern bioanalysis a good sample preparation and a hyphenated instrumentation are required. In pharmaceutical research companies the development of comprehensive bioanalytical methods is very important during the process of drug discovery and development. In addition the method validation has an important role in regulatory bioanalysis to ensure the quality of the applied method. Bioanalytical method validation is very important for supporting of new drug applications or biologics license applications.
The present review provides critical points to consider when carrying out bioanalytical method development and validation. Until now, different publications have been published with method validation trends but still lack of concise and comprehensive review for method validation providing specific requirements for conducting GLP nonclinical and clinical study sample analysis. In this paper required information for bioanalytical method development and method validation according to GLP guidelines will be presented. [1-12]

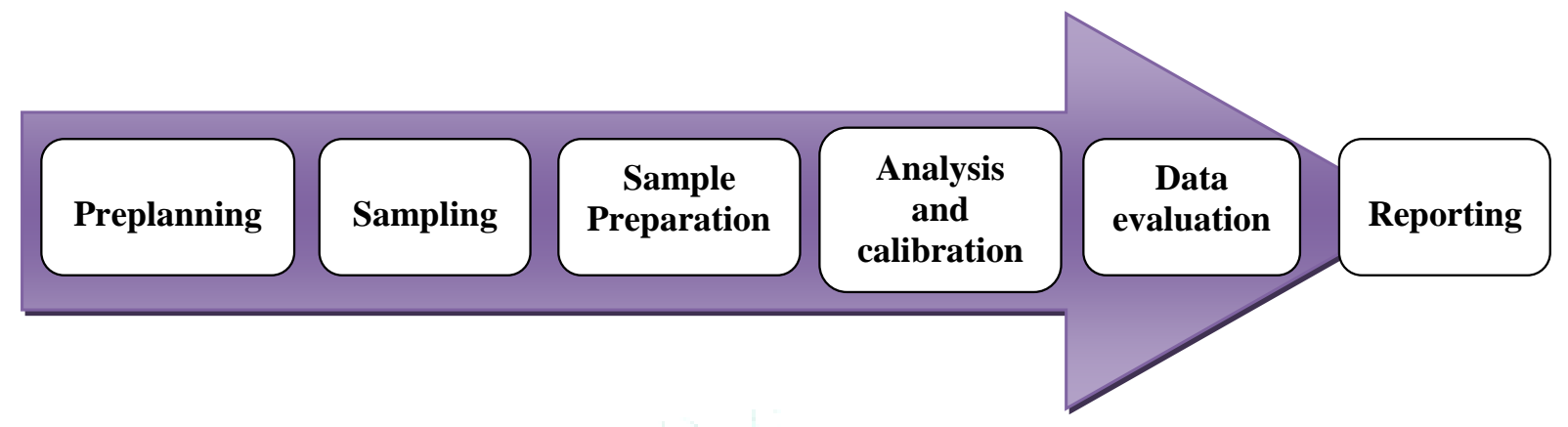

Figure N1:- Bioanalysis Procedure

\section{Need of Bioanalytical Method Validation}

$\checkmark \quad$ It is essential to used well-characterized and fully validated Bioanalytical methods to yield reliable results that can be satisfactorily interpreted.

$\checkmark$ It is recognized that Bioanalytical methods and techniques are constantly undergoing changes and improvements; they are at the cutting edge of the technology.

$\checkmark$ It is also important to emphasize that each Bioanalytical technique has its own characteristics, which will vary from analyte to analyte, specific validation criteria may need to be developed for each analyte.

$\checkmark$ Moreover, the appropriateness of the technique may also be influenced by the ultimate objective of the study. When sample analysis for a given study is conducted at more than one site, it is necessary to validate the Bioanalytical method(s) at each site and provide appropriate validation information for different sites to establish inter-laboratory reliability.

\section{Bio-analytical Method Development}

A bio-analytical method is a set of all of the procedures involved in the collection, processing, storage or storing and analysis of a biological matrix for an analyte. Analytical methods working for quantitative determination of drugs and their metabolites in biological fluids are the key determinants in generating reproducible and dependable data that in turn are used in the evaluation and elucidation of bioavailability, bioequivalence and pharmacokinetics.
Method development is a scientific procedure. It involves evaluation and optimization of the various stages of sample preparation, chromatographic conditions, detection and quantification.

\section{Separation and Detection by RP-HPLC}

High performance liquid chromatography (HPLC) is a powerful technique in bioanalysis. HPLC columns have a wide range of selectivity and therefore HPLC was applied for the separation of many drugs and metabolites in different matrices. The main type of detectors used in HPLC is UV-vis. HPLC-UV was applied for separation and determination of various analytes and matrices. UV detector is cost effective but can be used only for high analyte concentration $(\mu \mathrm{M}$ range), for small sample volumes and low concentration levels $(\mathrm{nM}, \mathrm{pM})$ the mass spectrometry is most useful detector. [14-18]

In bio-analytical method development various activities are carried out. Stages involved in method development using RP- HPLC are as follows:
$\checkmark \quad$ Literature search for drugs.
$\checkmark$ Identification of analytical technique and optimization
$\checkmark$ Reference standard preparation
$\checkmark$ Selection and optimization of extraction method
$\checkmark$ Optimization of HPLC method 
Literature Search for Drugs.

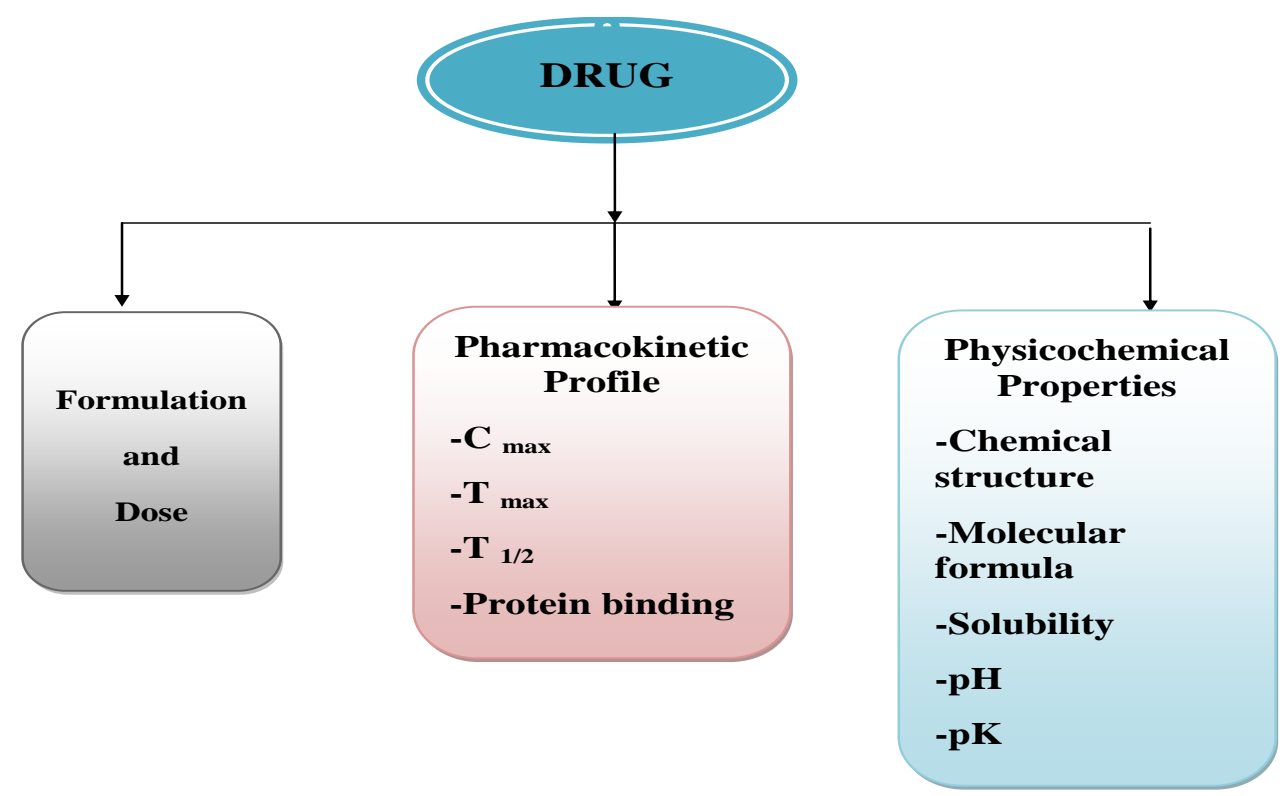

Figure 2:- Literature Search for Drugs

Selection of Appropriate Column and Mobile Phase:-

\author{
$\checkmark$ Mode of separation \\ $\checkmark$ Selection of appropriate column \\ $\checkmark$ Mobile phase \\ $\checkmark$ Role of pH, Buffer \\ $\checkmark$ Role of Temperature \\ $\checkmark$ Role of flow rate
}

\section{Established Extraction Techniques in Drug Bioanalysis}

Sample clean-up (sample preparation) is a primarily step before injection of complex matrices such as plasma, blood, urine and tissue into the analytical instruments. Regularly used sample preparation methods in many bioanalytical laboratories are protein precipitation (PPT), Liquid-liquid extraction (LLE), solid phase extraction (SPE). In the following sections the main sample preparation techniques will be discussed

\section{Liquid-liquid extraction (LLE)}

LLE is still an smart technique in sample preparation and it has been extensively used for the preparation of aqueous and biological samples. Basically, in liquid-liquid extraction an aqueous sample (e.g., plasma, urine) and an immiscible organic solvent are mixed to eliminate the analyte into the organic phase for injection into an analytical system. This method can provide good recovery and clean sample. LLE was used for the extraction of basic and acidic drugs from biological samples with high extraction recovery.

\section{Solid phase extraction (SPE)}

At the present time, SPE is the most well-known sample preparation method due to the high efficiency, cost-effective, high-reproducibility, relatively green and easy to operate and automate. SPE is beneficial such as separating and concentrating of trace analytes in biological samples. SPE is a straight forward method that uses a sorbent of 50-200 mg as cartridge to separate required analytes from a complex matrix. Recently, other formats of SPE have also been developed such as, flat disks and $\mu$ SPE. The nature and amount of the sorbent, loaded sample volume (with enough recovery), composition and volume of the washing and elution solutions (without loss of the analytes) are the effective parameters in SPE performance.

Solid Phase Extraction types and mechanism:

\section{A- Reversed-phase,}

Reverse Phase-SPE, (polar sample matrix includes nonpolar analytes using nonpolar sorbent). The used sorbents in this type are bonded silica (C4, C8, C18 and Ph, with $40 \mu \mathrm{m}$ particle size and $60 \AA$ pore size) and polymer sorbent as polystyrene can be used. In general the analytes are intermediate polar to nonpolar and hydrophobic interactions between analytes and nonpolar sorbent material occurred. This type of SPE is less selective compared to normal phase or ion-exchange SPE. The used elution solutions in RP-SPE are methanol, acetonitrile or mixed buffer/solvent. Typical applications in RP-SPE are drugs in biological fluids and environmental pollutants in water.

\section{B- Normal-phase}

Normal Phase- SPE (polar analytes in nonpolar matrices using polar sorbents). Typical sorbents in NP-SPE are silica with polar functional groups (Si-CN, Si-NH2, Si-Diol and pure silica). In NP, the retention mechanism is based on hydrogen bonding between analytes and sorbent.

\section{C- Ion exchange (anion and cation-exchange)}

SPE, for charged analytes in sample solution. Based on this method the anionic analytes (acidic drugs) can be isolated with quaternary amine bonded silica or Si-NH2 as anion exchange. For basic drugs, strong cation exchange, Si-SCX (propylsulfonic acid bonded) and week cation exchange, Si WCX (carboxy propyl phase) can be used for isolating the cationic analytes (basic drugs). Sulfonic acid bonded silica with $\mathrm{Na}+$ counter ion and carboxylic acid-bonded silica with $\mathrm{Na}+$ counter ion are common sorbents for SCX and WCX, respectively. Ion-exchange is the most selective method in SPE methodology 


\section{Protein precipitation (PPT)}

Precipitation is broadly applied for product recovery of biomolecules particularly proteins. Protein precipitation is appropriate for plasma or blood samples especially at high analyte concentration. Precipitation is generally induced by addition of a miscible organic solvent (acetonitrile, acetone or methanol), salt (aluminium chloride), metal ions (zinc sulphate) or by changing the sample $\mathrm{pH}$ to alter the nature of the solution (acids such as trichloroacetic, perchloric, met phosphoric and tungstic). Protein precipitation as a fast and simple extraction approach can be applied for both hydrophilic and hydrophobic compounds. In some cases (for extraction of some drugs and metabolites) the protein precipitation method can be followed by LLE or SPE in order to achieve higher efficiency.

\section{Microextraction techniques}

The use of microextraction techniques in bioanalysis has been gained attraction due to achieve high throughput sample preparation, to handle small sample volumes and to decrease solvents consuming. Last two decades new microextraction techniques such as solid-phase microextraction (SPME), stir-bar sorptive extraction (SBSE), microextraction by packed sorbent (MEPS) and packed tips were introduced in bioanalysis.

\subsection{Solid phase microextraction (SPME)}

SPME was introduced in the year of 1989 and has been accepted as a powerful solvent-free extraction technique especially for GC. SPME has been accepted as a straight forward and automated method in environmental analysis and lesser amount in bioanalysis. Commonly in SPME, a fused silica fiber is coated with a stationary phase. The equilibrium in SPME is established by exposing fiber with the analyte in matrix. SPME has two general sorts; direct extraction of analytes with concentration of the fiber into the sample, or utilizing headspace to extract volatile compounds from fiber surface which are partitioned between gaseous and liquid phases. To have an efficient SPME process some parameters such as; temperature, $\mathrm{pH}$, salt concentration, stirring rate, the equilibrium constant and equilibration time need to be optimized. SPME is a practical technique but still suffering from some drawbacks such as; short fiber lifetime and low sensitivity of analytes in complex matrices.

\subsection{Stir bar Sorptive extraction (SBSE)}

Stir bar sorptive extraction (SBSE) was introduced in the year of 1999 by Sandra et al. and based on the similar principle of SPME. In this technique, a magnetic stirring bar of quartz coated with polydimethylsiloxane (PDMS) is used. Compared to SPME the SBSE has more coated polymer $(>50$ times) and so higher extraction efficiency is obtained using SBSE compared to SPME. SBSE is cost effective technique and it is commonly used in environmental applications but it was also applied in bioanalysis for the extraction of some drugs and metabolites from biological fluids.

\subsection{Microextraction by packed sorbent (MEPS)}

MEPS was invented in 2004 as a novel, simple, fast, costeffective, readily automated and green samples preparation method. MEPS is $\mu$-SPE technique that can be connected online to GC or LC systems. In MEPS the sorbent is fitted in a liquid handling microsyringe as a plug with low-voidvolume. When the sample is drawn through the syringe, the analytes are adsorbed onto the solid phase. The sorbent then is washed with water and finally the analytes were absorbed by suitable solvent.
Microextraction packed sorbent steps are similar to SPE and needs to consider some solutions (based on the analyte structure and polarity) for conditioning (100-200 $\mu \mathrm{L}$ acidic water with a small amount of solvent such as methanol (20\%), loading $(4 \times 100 \mu \mathrm{L})$, washing $(100 \mu \mathrm{L}$ of $0.1 \%$ formic acid in water or with $5 \%$ methanol in water) and eluting (20 to $50 \mu \mathrm{L}$ methanol or acetonitrile) sections. Different sorts of sorbents can be used in MEPS such as; C18, C8, C2, C8/SCX, SCX, SAX, imprinted polymers and silica. MEPS has been applied for determination of different drugs and metabolites in different types of biological fluids.

\subsection{Packed tips}

Recently, the packed sorbents (silica and monolithic based sorbents, imprinted polymers) in a single tip or 96-tips (monolithic) have been developed with great efficiency and selectivity in bioanalysis. The extraction steps and process are almost similar to SPE and MEPS. Monolithic packed 96tips is a clean, fast, high-throughput and semi-automated sample preparation and can handle small sample volumes. [13]

Table 1. Bioanalytical method development and validation strategies

\begin{tabular}{|c|c|}
\hline Subject & Requirements / Activity \\
\hline Analyte & $\begin{array}{l}\text { Chemical structure } \\
\text { Chemical properties } \\
\text { Analyte purity }\end{array}$ \\
\hline $\begin{array}{c}\text { Sample } \\
\text { preparation }\end{array}$ & $\begin{array}{l}\text { Nature and property of the matrix } \\
\text { Matrix stability } \\
\text { Choose of suitable extraction method } \\
\text { Extraction recovery }\end{array}$ \\
\hline $\begin{array}{c}\text { Method } \\
\text { validation }\end{array}$ & $\begin{array}{l}\text { Calibration curve with QC samples } \\
\text { Accuracy } \\
\text { Precision } \\
\text { Selectivity } \\
\text { Matrix effect } \\
\text { Carry-over } \\
\text { Stability (short and long term) }\end{array}$ \\
\hline
\end{tabular}

\section{Bio-Analytical Method Validation}

Method validation can be defined as "Establishing documented evidences, which provides a high degree of assurance that a specific method or activity will consistently produce a desired result or product meeting its predetermined specifications and quality characteristics". Bio- analytical method validation includes all of the procedures that demonstrate that a particular method used for quantitative measurement of analyte in a given biological matrix, such as blood, plasma, serum and urine is reliable and reproducible for the intended use.

\section{Types of Method Validation}

\section{Full Validation}

Full validation is important when developing and implementing a bio-analytical method for the first time and is also important for a new drug entity. A full validation of the revised assay is important if metabolites are added to an existing assay for quantification.

\section{Partial Validation}

Partial validation is modification of already validated bioanalytical method. Partial validation can range from as minute as one intra-assay accuracy and precision determination to a practically full validation.

Classic bio-analytical method changes that drop into this category include, 
- Change in analytical methodology (e.g. change in detection systems)

- $\quad$ Change in sample process in procedures

- Change in anticoagulant in harvesting biological fluid

- Change in matrix within species (e.g. human plasma to human urine) or

- $\quad$ Change in species within matrix (e.g. rat plasma to mouse plasma)

\section{- Change in relevant concentration range}

Selectivity demonstration of an analytes in the presence of concomitant medications or of specific metabolites.

\section{Cross Validation}

Cross-validation is a comparison of validation parameters when two or more bio-analytical methods are used to generate data within the same study or across different studies. An example of cross validation would be a situation where an original validated bio-analytical method serves as the reference and the revised bio-analytical method is the comparator. When sample analysis within a single study are conducted at more than one site or more than one laboratory, cross validation with spiked matrix standards and subject samples should be conducted at each site or laboratory to create inter laboratory reliability.[19]

\section{Various Parameters involved in validation}

Linearity

\section{Accuracy \& Precision}

A. Precision Intra-day or within batch

B. Precision Inter-day or between batch

System suitability

Stability
A. Stock Solution Stability
B. Bench Top Stability
C. Freeze-Thaw Stability
D. Short term Stability at $-20^{\circ} \mathrm{C}$
E. Long Term Stability

Recovery studies

\section{Matrix Effect}

\section{Linearity}

Calibration (standard) curve is the relationship between instrument response and known concentrations of the analytes. A calibration curve should be prepared in the same biological matrix as the samples in the proposed study by spiking the matrix with known concentrations of the analytes. The number of standards used in constructing a calibration Curve will be a function of the anticipated range of analytical values and the nature of the analytes/response relationship.

\section{Precision and Accuracy}

The accuracy of an analytical method describes the imminence of mean test results obtained by the method to the true value (concentration) of the analytes. Accuracy should be measured using a minimum of five determinations per concentration. The precision of an analytical method describes the nearness of individual measures of an analytes when the procedure is applied repeatedly to multiple aliquots of a single homogeneous volume of biological matrix. Precision be supposed to be measured using a minimum of five determinations per concentration. Precision is expressed as the coefficient of variation (CV). Precision should be demonstrated for the LLOQ, low, medium and high QC samples. [20]

\section{System Suitability:}

System Suitability is the ability of an analytical method to differentiate and quantify the analytes in the presence of other components in the sample. The selectivity of the method was evaluated by analyzing six replicates of plasma samples spiked at LLOQ (Lower Limit of Quantification).

\section{Stability}

Drug stability in a biological fluid is a role of the storage conditions, the chemical properties of the drug, the matrix, and the container system. Stability evaluation is done to show that the concentration of analytes at the time of analysis corresponds to the concentration of the analytes at the time of sampling.

\section{A) Stock Solution Stability}

The stability of stock solutions of drug and the internal standard should be evaluated at room temperature for at least six hours. If the stock solutions are refrigerated or frozen for the relevant period, the stability should be recognized. After completion of the desired storage time, the stability should be tested by comparing the instrument response of stored sample with that of freshly prepared solutions.

\section{B) Bench Top Stability}

Bench top stability is to assess the stability of analytes in biological fluids over a period of time during which the sample are expected to be kept on bench while processing.

\section{C) Freeze Thaw Stability}

Analytes stability should be determined after three freeze and thaw cycles. At least three aliquots at each of the low and high concentrations should be stored at the intended storage temperature for 24 hours and thawed unaided at room temperature. When the sample completely thawed, the samples should be refrozen for 12 to 24 hours under the same conditions. The freeze-thaw cycle should be repeated two more times, and then analyzed on the third cycle. This Freeze-Thaw quality control samples were quantified against the freshly spiked calibration curve standards of concentration range equivalent to that used for the calculation of precision and accuracy.

\section{D) Short Term Temperature Stability}

Three aliquots of each of the low and high concentrations should be thawed at room temperature and kept at this temperature from 4 to 24 hours (based on the expected duration that samples will be maintained at room temperature in the intended study) and analyzed.

\section{E) Long Term Temperature Stability}

The storage time in a long-term stability evaluation should exceed the time between the date of first sample collection and the date of last sample analysis. Long-term stability should be determined by storing at least three aliquots of each of the low and high concentrations under the same conditions as the study samples. The volume of samples should be sufficient for analysis on three separate occasions. The concentrations of all the stability samples should be 
compared to the mean of back-calculated values for the standards at the appropriate concentrations from the first day of long-term stability testing.

\section{Recovery Study}

The recovery of analytes in an assay is the detector response obtained from an amount of the analytes added and extracted from the biological matrix, compared to the pure authentic standard. Recovery pertains to the extraction efficiency of an analytical method within the limits of variability. Recovery of the analytes need not be $100 \%$, but the extent of recovery of an analytes and of the internal standard should be consistent, precise, and reproducible. Recovery experiments should be performed by comparing the analytical results for extracted samples at three concentrations (low, medium, and high) with unextracted standards that represent $100 \%$ recovery. [21-23]

\section{Matrix Effect}

Matrix effect is defined as the effect of co-eluting residual matrix component of biological sample on the ionization of target. There are two types of the matrix effect e.g. absolute matrix effect and relative matrix effect. Absolute matrix effect is defined as difference between response of equally concentrated analyte in solvent and in matrix extracts. Two types of absolute matrix effect one is ion suppression which is more often observed and other is ion enhancement. Relative matrix effect is defined as the variation of absolute matrix effect between several lots of same matrix. Matrix effect may cause decrease or increase in sensitivity over the time, increased baseline, imprecision of result, retention time change and chromatographic peak tailing. Some sample preparation procedures have been of tremendous significance in reducing matrix effect. As demand for assay sensitivity rises because of evaluation increase in potent drug and which can be detected in low concentration. Selection of sample preparation to minimize matrix effect is heavily determined by molecular analysis and specimen type. Volume of reagent, flow rate, and various protocol parameters of matrix reduction should be compatible with analysis. In the number of cases, matrix components which might cause ionization enhancement or suppression are removed during sample cleaning process. Many method used for sample clean up most simple method is protein precipitation method. Other method used is liquid extraction and solid phase extraction method. Matrix effect can be caused by organic and inorganic substances present, amines, urea, carbohydrates etc. The CV of the IS-normalized matrix factor (MF) calculated from the 6 lots of matrix should not be greater than $15 \%$. This determination should be done at a low and at a high level of concentration (maximum of 3 times the lower limit of quantification (LLOQ) and close to the upper limit of quantification (ULOQ). The overall coefficient of variation (CV) calculated for the concentration should not be greater than $15 \%$. [24-26]

\section{Conclusion}

In this review, the basic required concepts and definitions in bioanalytical method validation were presented and discussed. SPE and LLE are most used sample preparation techniques in bioanalysis. Huge progress has been done in SPE technology such as new SPE format and new selective sorbents. In addition progress in the automated systems for sample preparation is expected to meet the need for high sample throughput. In addition progress in the automated systems for sample preparation is expected to meet the need for high sample throughput. Regulatory bioanalysis has an essential role in pharmaceutical research and development.

\section{References:-}

1. D. Zimmer, Introduction to quantitative liquid chromatography-tandem mass spectrometry (LC-MS-MS), Chromatographia 57 (2003) 325-332.

2. J. Schuhmacher, D. Zimmer, F. Tesche, V. Pickard, Matrix effects during analysis of plasma samples by electrospray and atmospheric pressure chemical ionization mass spectrometry: practical approaches to their elimination, Rapid Commun. Mass Spectrom. 17 (2003) 1950-1957.

3. E. Stokvis, H. Rosing, J.H. Beijnen, Stable isotopically labeled internal standards in quantitative bioanalysis using liquid chromatography/mass spectrometry: necessity or not?, Rap. Com. Mass Spec. 19 (2005) 401-407.

4. T.M. Annesley, Ion suppression in mass spectrometry, Clin. Chem. 7 (2003) 1041-1047.

5. B.K. Matuszewski, M.L Constanzer, C.M. Chavez-Eng, Strategies for the Assessment of Matrix Effect in Quantitative Bioanalytical Methods Based on HPLC-MS/MS, Anal. Chem. 75 (2003) 3019-3030.

6. R. Weaver, R.J. Riley, Identification and reduction of ion suppression effects on pharmacokinetic parameters by polyethylene glycol 400, Rap. Com. Mass Spec. 20 (2006) 2559-2564.

7. A. V. Eeckhaut, K. Lanckmans, S. Sarre, I. Smolders, Y. Michotte, Validation of bioanalytical LC-MS/MS assays: Evaluation of matrix effects, J. Chromatogr. B, 877 (2009) 2198-2207.

8. M. Ahnoff, A-C. Nyström, F. Schweikart, A. Ekdahl, Matrix effect explained by unexpected formation of peptide in acidified plasma, Bioanalysis 7 (2015) 295-306

9. [12] M.W.J. van Hout, H.A.G. Niederländer, R.A. de Zeeuw, G.J. de Jong, Ion suppression in the determination of clenbuterol in urine by solid-phase extraction atmospheric pressure chemical ionisation ion-trap mass spectrometry, Rap. Com. Mass Spec. 17 (2003) 245-250.

10. D. Remane, M.R. Meyer, F.T. Peters, D.K. Wissenbach, H.H. Maurer, Fast and simple procedure for liquid-liquid extraction of 136 analytes from different drug classes for development of a liquid chromatographic tandem mass spectrometric quantification method in human blood plasma, Anal. Bioanal. Chem. 397(2010) 2303-2314.

11. O. González, M.E. Blanco, G. Iriarte, L. Bartolome, M.I. Maguregui, R.M. Alonso, Bioanalytical chromatographic method validation according to current regulations, with a special focus on the non-well defined parameters limit of quantification, robustness and matrix effect, J. Chromatogr. A 1353 (2014) 10-27.

12. A.R. Buick, M.V. Doig, S.C. Jeal, G.S. Land and R.D. McDowall, Method validation in the bioanalytical laboratory, J. Pharm. Biomed. Anal. 8 (1990) 629-637.

13. Mohamed Abdel-Rehim, Bioanalytical method development and validation: critical concepts and strategies,2016

14. S. Kamaruzaman, M.M. Sanagi, S. Endud, W.A. Wan Ibrahim, N. Yahaya, MCM-41 solid phase membrane tip extraction combined with liquid chromatography for the determination of non-steroidal anti-inflammatory drugs in human urine, J. Chromatogr. B 940 (2013) 59-65.

15. G.M. Ben-Hander, A. Makahleh, B. Saad, M.I. Saleh, Hollow fiber liquid phase microextraction with in situ derivatization for the determination of trace amounts of metformin hydrochloride (anti-diabetic drug) in biological fluids, J. Chromatogr. B 941 (2013) 123-130.

16. H. Karim, M.L. Appell, A. Fotoohi, Comparison of three methods for measuring thiopurine methyltransferase activity in red blood cells and human leukemia cells, J. Chromatogr. B 939 (2013) 80-85.

17. J. Tonic-Ribarska, A. Haxhiu, Z. Sterjev, G. Kiteva, L. Suturkova, S. TrajkovicJolevska, Development and validation of a bioanalytical LC-UV method with solid-phase extraction for determination of valproic acid in saliva, Acta Pharm. 62(2012) 211-20.

18. R. D. ValérioI, R. M. SilvaI, H. P. Cunhal, M. C. A. LimaII, S. L. GaldinoII, I. R. PittaII, M. B. S. Maia, Development and validation of an LC-UV method for quantification of a new thiazolidinedione (LPSF/AC-23) with antitumor activity in rat plasma, J. Braz. Chem. Soc. 23 (2012) 2104-2113.

19. Guideline on bioanalytical method validation, 21 July 2011 
EMEA/CHMP/EWP/192217/2009 Rev. 1 Corr. 2,Page no.1011

20. International Conference on Harmonization of Technical Requirements for Registration of Pharmaceuticals for Human Use ICH Harmonized tripartite guideline Validation of analytical procedures: Text and Methodology Q2 (R1) 6 November 1996.

21. Vinod P. Shah, Bioanalytical Method Validation, A Revisit with a Decade of Progress, Pharmaceutical Research, December 2000,Volume 17, Issue 12, pp 1551-1557

22. David Watson G., Pharmaceutical Analysis (3 rd Ed., Churchill Livingstone, London: Harcourt Publishers limited, Essex CM 20 2JE, 2012
23. Current Good Manufacturing Practices for finished Pharmaceuticals, 21 CFR, Parts 210 and 211, US Food and Drug Administration.

24. Willard HH, Merritt Jr LL, Dean JA, Settle Jr FA. Instrumental methods of analysis.CBS Publ.; 1981:19-90.

25. McDowall RD. The role of laboratory information management systems LIMS in analytical method validation. Anal Chim Acta 2007; 54:149-58.

26. Wieling J, Hendriks G, Tamminga WJ, Hempenius J, Mensink $\mathrm{CK}$, Oosterhuis B, et al. Rational experimental design for bioanalytical methods validation. Illustration using an assay method for total captopril in plasma. J Chromatogr A 1996; 730(12):381-94 